\title{
Signs Workshop: the Importance of Natural Gestures in the Promotion of Early Communication Skills of Children with Developmental Disabilities
}

\author{
Ana Margarida P. Almeida ${ }^{1}$, Teresa Condeço ${ }^{2}$, Fernando Ramos ${ }^{1}$, Álvaro Sousa ${ }^{1}$, \\ Luísa Cotrim ${ }^{2}$, Sofia Macedo ${ }^{2}$, Miguel Palha ${ }^{2}$ \\ ${ }^{1}$ Department of Communication and Arts, University of Aveiro, Campus de Santiago, 3810- \\ 193 Aveiro \\ 2 Differences, Child Developmental Centre, Centro Comercial da Bela Vista, Av. Santo \\ Condestável, Loja 32, Via Central de Chelas, 1950-094 Lisboa
}

\begin{abstract}
This article emphasises the importance of natural gestures and describes the framework and the development process of the "Signs Workshop" CD-ROM, which is a multimedia application for the promotion of early communication skills of children with developmental disabilities. Signs Workshop CD-ROM was created in the scope of Down's Comm Project, which was financed by the Calouste Gulbenkian Foundation, and is the result of a partnership between UNICA (Communication and Arts Research Unit of the University of Aveiro) and the Portuguese Down Syndrome Association (APPT21/Differences).
\end{abstract}

Keywords: language and communication skills, augmented communication systems, total communication (simultaneous use of signs and language), multimedia production.

\section{Introduction}

The Down's Comm Project, fully financed by the Calouste Gulbenkian Foundation, is the result of a partnership between the Communication and Arts Research Unit of the University of Aveiro (UNICA) and the Portuguese Down Syndrome Association/Differences (APPT21).

This project's main objective was to research (and translate into an interactive multimedia application) examples of natural gestures from the Portuguese culture, in order to ensure the expansion and flexibility of its use by parent, educators and therapist who care for children with developmental disabilities, particularly children with difficulties in the development of speech.

Thus, it is intended to help in the creation of support strategies in order to promote the early stage of sign utilization, understood as a critical link which ensures the transition from the pre-verbal communication stage to the spoken language stage [1]. 
This research project resulted in the production of the Signs Workshop CD-ROM, which is clearly directed to a population target characterized by a developmental disability and speech difficulties from birth, and is assumed to be a working support tool for parents, educators and therapists who work for the promotion of communication and interactions skills.

Within this framework, the main objectives of the Signs Workshop CD-ROM are: to promote the development of language and communication skills, at the pre-verbal stage; to enable learning and access to different natural gestures, commonly used in the Portuguese day to day culture; to provide, for each gesture, a set of information in several formats (text, sound, image, video), enabling various and personalized searches.

\section{Communication Skills and Sign Communication Systems}

Children with developmental difficulties, especially those with Down Syndrome, present changes in the development and use of language, with particular emphasis at the speech development level [2] [3] [4]. Hence, the subsequent difficulties to communicate surface since the pre-verbal stage, which result in a general tendency to show passivity in communicating and in a low ability to take initiative towards interacting with other individuals.

Therefore, these children's abilities to express themselves verbally are frequently inferior to those of understanding [5] [6] [7]. In some cases, children may not even be able to speak comprehensibly as the result of a deep developmental disability, great hearing loss or great motor or neuro-muscular difficulties [5] [3].

Some studies suggest that, in these cases, the bridge or link between the pre-verbal communication stage and the spoken language stage may be ensured by an early use of signs, which should thus precede the introduction of verbal signs. This should also be the time to promote a guided access and use of communication media [1] [8] [5] [9] [10] [11].

It is within this framework that Augmented Communication Systems are proposed. These systems provide an important support to message expression by the individuals who present speech difficulties, either temporary or permanent. In this context, it is important to identify Augmented Communication with all systems which supplement, support or substitute speech.

The Sign Communication Systems or Sign Language Systems, when organized in symbolic or coded signs, are examples of the Augmented Communication Systems frequently used [12]. In effect, in the specific case of children with Trisomy 21, the Augmented Communication System designated as Total Communication (simultaneous use of signs and language) is intensively used as a temporary system of transition during the early stages of speech development. This transition temporary system is particularly appropriate to children who did not initiate speech exercise around the 12-18 months of age, and who, in consequence, present signs of frustration by their incapacity of being understood by parents, siblings or other individuals [12].

The application of this Total Communication System highlights, therefore, the importance of using signs as a support to various communication functions, which 
would not be possible any other way: request, make questions, ask for information, express their own experiences or play.

From the viewpoint of parent-child interaction, the use of signs also enhances the communication processes and their adaptation to the child's abilities: the use of signs that represent words, by the parents, occurs in contexts where they speak slower, use shorter sentences and, probably, put into emphasis the words that are said and signalled [13]. Another significant feature of simultaneous signalling and speech is the fact that parents assure eye contact with the child while communicating with them. Parents are, thus, in a better position to observe their children's behaviour and responses, and react accordingly [13]. The simultaneous use of visual and audio communication forms may, then, facilitate information gathering and thus enhance cognitive skills [13].

\section{Portuguese Cultural Daily Life Signs Standardization}

The research made by the APPT21/Diferenças team, with the objective of standardizing the signs to include in the CD-ROM, was gathered among Portuguese population (continent and islands) with diagnosed Down Syndrome, who, in an early age, uses or has used, at some point, Total Communication (simultaneous use of natural gestures and speech).

To achieve its goal, the team produced an inventory built from existing national and international approaches: American Sign Language, Portuguese Sign Language Book, Portuguese Makaton, "See \& Say" of the Sarah Duffen Centre of Portsmouth, and also information gathered from the experience of the APPT21/Differenças therapist team [14] [15] [16].

The mentioned inventory (figure 1) was sent to parents whose children have Down Syndrome and to therapists and educators who work with this population. They were asked to identify the signs they use in daily life and to suggest others for the presented concepts. They were also asked to provide information of other signs or concepts that were not included in the inventory.

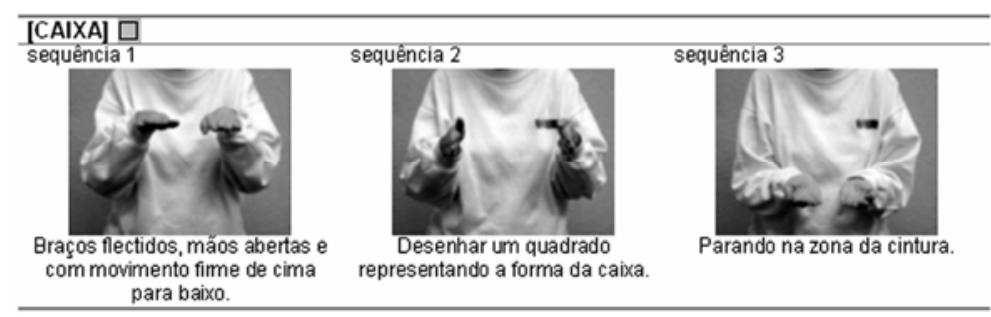

Fig. 1. Example of a sign in the inventory.

After analyzing the returned inventories, the team proceeded with the data treatment: the suggested signs were added to the initial inventory, and others were modified according to the parents and therapist's recommendations. At the end of the research, the team was able to gather and standardize 184 signs. 


\section{Specification and Development of the Multimedia Application}

Once defined the objectives and contents for the application, functional requirements specification proceeded: language selection (Portuguese, English, Spanish); sign search (alphabetically, open field and by category); user profile creation (in order for the user to store the favourite signs); user profile registration (by inserting a login); user profile edition (user can remove, add or print favourite signs); sign search according to profile (search for stored signs); sign search display (includes the sign designation, text and audio description, video and graphic display sequences); printing sign search display (includes the sign designation, text description and graphic display sequences); signs in context (permits sign viewing from the same context of the selected sign); adding signs to user profile (adds the selected sign to the user profile); help section (user support for CD-ROM interaction and navigation).

\section{Functional and Technical Design}

After specifying the conceptual model which resulted from the identification of the above mentioned functional requirements, the CD-ROM production was taken to the next level - the functional design [17] - and a paper script was created. This allowed not only having a clearer view of all functionalities to include in this application, but also facilitated communication among the design team.

At this point, it was also initiated a technical viability study, with the objective of beginning the parallel and iterative development of the Technical Design [17], as well as to determine which technology would be more suitable for the production of this application. In order to ensure the communication between the sign's database and the application's interface, the team decided to use Microsoft Access and Macromedia Director (with Xtras Datagrip, BuddyAPI and FileIO).

As soon as the script and the technical viability study were validated, a prototype was developed. This prototype assumed a crucial role for the analysis, discussion and correct definition of the functional objectives of the CD-ROM.

Simultaneous to the prototype creation, the team started on the communication design studies: colour schemes, symbols/icons and graphic interfaces. The challenge of providing adequate responses to specific social, technological and communicative conditions, combined with the need to create a user-friendly interface with a fast learning rate, lead to the specification of three key concepts which sustain the communication design: space, lightness and order. Having that outlined, and in an attempt to accomplish a clear and efficient visual scheme, three main colours were defined: white, green and grey.

The symbol (figure 2), based upon a line sketch and with a clear hand-like characteristic, represents two hands of a character with an open smile, and intends to create an environment of involvement. The emotional aspect of this illustration meets the sentimental feature of this application, balancing a more rational side manifested in all structural and formal aesthetic of the CD-ROM: the green colour (fresh and bright) offers it lightness and joy, and it is used as a distinguishing shade, allowing to weaken the grey's neutrality, which was chosen for the information elements. 


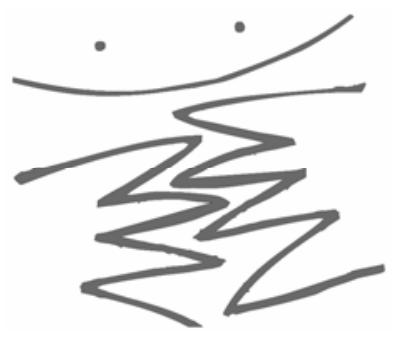

Fig. 2. Symbol .

As far as interface designing is concerned, a grid/layout was created allowing the establishment of structuring areas (navigation and contents) and of a set of navigation icons, which in coherence with the formal language represent the main functions and ensure an immediate viewing of all accessible fields (figure 3 ).

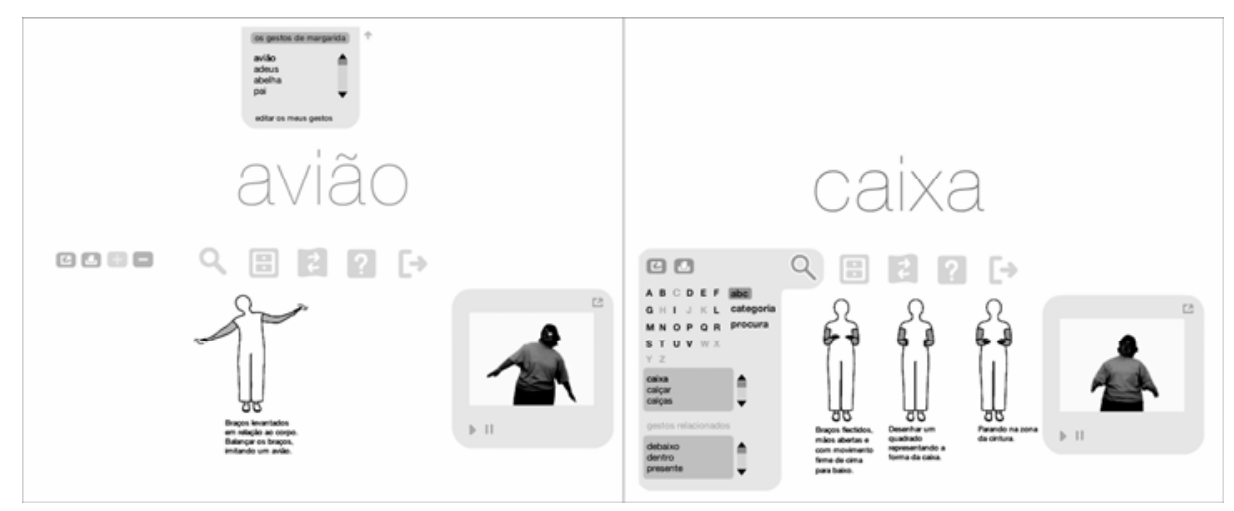

Fig. 3. Graphic Interfaces.

\section{Content Creation and Edition}

The design of the sign's graphic representations (in different image sequences) was made from a graphic simplification and systematization of the human figure (figure 4). This figure, reduced to a contoured line, is somewhat between a realistic representation of the person who signals and a schematic representation, and adds a more rational illustrative feature to the application. From the mentioned graphic organization, was then possible to sketch the different sequences of the graphic representations of the 184 signs (figure 4). 

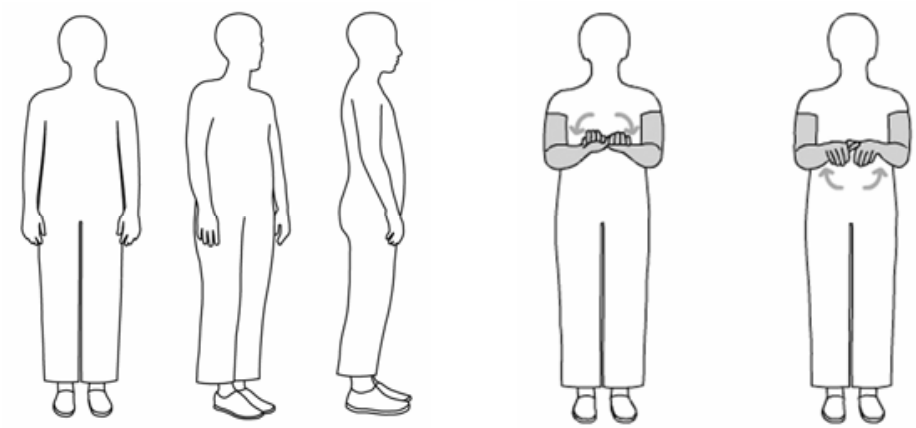

Fig. 4. Graphic systematization of the human figure and graphic representation sequence.

In what concerns video contents, which show the real representation of a sign, 184 signs were captured in the APPT21 facilities: young boys and girls with Trisomy 21 executed the signs. During the process of recording and editing, the team gave special attention to the issues of light, saturation and contrast. With the objective of eliminating backgrounds (the intention was to obtain just the body outline over the application's white background), it was necessary to equalize the levels of light and to apply background removal filters (figure 5).

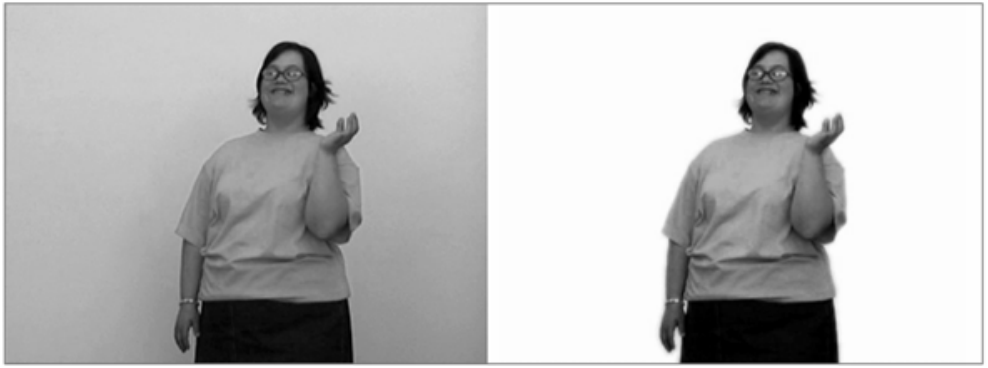

Fig. 5. Video editing (background removal)

Fulfilling the intention of integrating descriptive audio cuts of the signs, the team recorded and edited the 184 signs in the CD-ROM's three available languages: Portuguese, English and Spanish. The purpose of including audio features in the application is to support the video footage and the visual sign representations, in order to reinforce sign learning. Therefore, for each sign three distinct audio cuts can be differentiated: the sign designation, the sign's complete text description (for video support), the sign's partial text description (for supporting the graphic representation sequences). 


\section{Application Editing and Distribution}

Once the application was concluded, the next stage was initiated: development of broadcast, distribution and support tools. To this end, a support website was created (http://www.ca.ua.pt/dc), as well as an e-mail account to establish contact lines and support to the CD-ROM's end-users (oficinadosgestos@ca.ua.pt).

The CD-ROM editing process (equally financed by the Calouste Gulbenkian Foundation) included the design of the packages and production of the 1500 copies, currently available for purchase at the APPT21/Differenças.

The package design process, CD-ROM label (figure 6) and user manual followed the same graphic elements developed by the design team, in order to bolster the visual identity of the product.

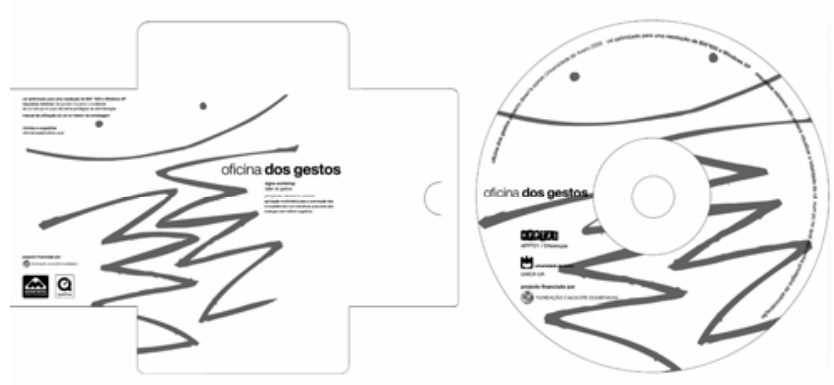

Fig. 6. CD-ROM's Package and Label

\section{Case Studies Short Description}

After the Signs Workshop CD-ROM development, many children with developmental disabilities have started using it, within the context of their personalized Early Intervention Programmes.

These programmes aim at promoting early communication skills, cognition and motor development and include the use of natural gestures as a Total Communication System. The familiar context is the main focus of this intervention: both family members and the child are introduced to the natural use of gesture as an effective support of the spoken word.

Early Intervention Programmes are structured in weakly sessions in which the psychologist, responsible for introducing the previous selected gestures, works with the child and the caregiver (usually, the mother or the father). Several activities and strategies that stimulate communication development are established for each weakly session and the caregivers are taught how and when to perform the gestures, so that they can use them effectively. Caregivers are also responsible for teaching the gestures to other family members and to kindergarten staff. 


\section{Case 1}

Case 1 is a 21 months old boy with Down Syndrome. He's on the Early Intervention Programme since 3 months old. Natural gestures were introduced at 4 months old and he is now able to express his needs and desires, like asking for food, toys or a particular kind of game. The child has already a repertoire of 20 gestures and is already able to "respond" to questions like "What's this?" or "What are you doing?".

He stays at home with his mother, so the 20 gestures that are now part of his repertoire are used with the closer family, especially with his 4 years old sister who motivates and helps him to perform the natural gestures.

\section{Case 2}

Case 2 is a 3 years old boy with Down Syndrome that is also on the Early Intervention Programme. He was introduced to the natural gesture since 10 months old and has now a repertoire of 30 natural gestures.

This child uses natural gestures at home, with his mother, and at kindergarten to ask for some particular kind of food, to go to the bathroom, or "respond" to the caregiver.

Gestures examples used by case 1 and case 2 children

Both case 1 and case 1 children use different gestures from the Signs Workshop CDROM as Eat, Drink, Dog, Cow, Banana and Orange, presented in Figure 7.

\begin{tabular}{|l|l|l|}
\hline Gesture Video frame & $\begin{array}{l}\text { Graphic } \\
\text { representation } \\
\text { sequence }\end{array}$ & $\begin{array}{l}\text { Text description } \\
\text { The closed hand } \\
\text { approaches the mouth } \\
\text { as if placing an } \\
\text { imaginary foodstuff in } \\
\text { it. The movement is } \\
\text { repeated several times. }\end{array}$ \\
\hline Drink & $\begin{array}{l}\text { The hand grasps an } \\
\text { imaginary glass, the } \\
\text { thumb close to the } \\
\text { mouth. } \\
\text { The hand tilts further } \\
\text { back towards the } \\
\text { mouth. }\end{array}$ \\
\hline
\end{tabular}




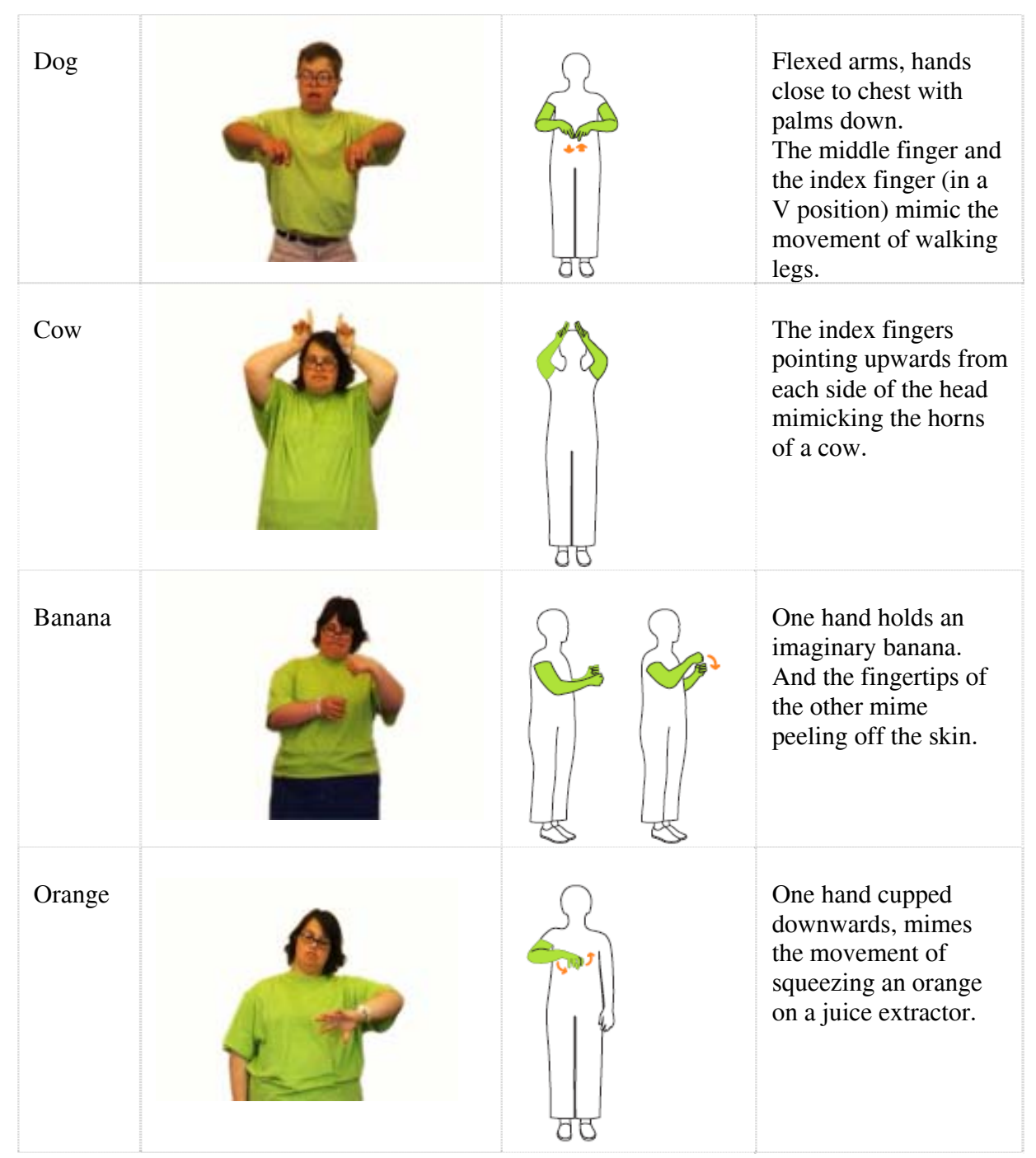

Fig. 7. Gestures examples.

\section{Conclusions}

The development process of the Signs Workshop CD-ROM was frame worked within an extended methodological model, in which not only the specification and conception of a multimedia software was valued, but mainly its adequate application to the individual, social and environmental particularities of its target population. 
The intensity and richness of the constant interactions with the end-users during the development process, enabled the accomplishment of the inter-disciplinary features that characterize this project, as well as widen work methods directed to the permanent contact with the natural environments of the target-group and, in consequence, develop suitable solutions to its specific universe.

By widely distributing this multimedia application, it is intended to make a positive contribution to the consolidation of knowledge on natural human signs within the context of Portuguese culture and provide to the education community a set of extended natural gestures, adequate to the development characteristics of Portuguese children with development disabilities.

\section{Acknowledgments}

The authors would like to thank to the Calouste Gulbenkian Foundation for the financial support; to the scholarship students Margarida Girão, Catarina Melo e Silva and Ana Filipa Gomes for the constant commitment and dedication to this project; to the young Teresa Palha and Henry Turquin (and families) for the participation in the video footage; to CEMED (Multimedia and Distant Learning Centre of the University of Aveiro) for the support in digital treatment and video editing; to João Miguel Lopes, José Leite, Laura Astorgano and Lina Hayek for collaborating in audio recording; to Manuela Castro, Maria João Santos and Rocio Bobadilha for the support in the translations; to Rui Pereira, Tiago Almeida, Pedro Almeida and Diogo Valente for the technical support; and to Fernando Ferreira for support in the inventory development.

\section{References}

1. Buckley, S. and Bird, G., "Teaching Children with Down's syndrome to Read", in: Down's Syndrome Research and Practice, The Journal of Sarah Duffen Centre, University of Portsmouth, Volume 1, Number 1, February, 1993, pp. 34-39.

2. Pueschel, S. M.., "Visual and Auditory Processing in Children with Down Syndrome", in: Nadel, L. (Ed.) The Psychobiology of Down Syndrome. Cambridge: National Down Syndrome Society, Library of Congress, Mit Press, 1988, p. 199-216.

3. Wishart, J. G., "Early Learning in Infants and Young Children with Down Syndrome", in: Nadel, L. (Ed.) The Psychobiology of Down Syndrome. Cambridge: National Down Syndrome Society, Library of Congress, Mit Press, 1988, p. 1-50.

4. Wishart, J. G. "Cognitive Development in Young Children with Down Syndrome: development strengths, development weaknesses", in: Down Syndrome in the 21st Century: 1st Biennial Scientific Conference on Down Syndrome, Down Syndrome Research Foundation and Resource Centre, 1998. Online: http://www.altonweb.com/cs/down syndrome/ wishart.html. Accessed on: 19-01-01.

5. Kumin, L. Communication Skills in Children with Down Syndrome, A Guide for Parents. Bethesda: Woodbine House, 1994. 
6. Miller, J. F., "The Development Asynchrony of Language Development in Children with Down Syndrome”, in: Nadel, L. (Ed.) The Psychobiology of Down Syndrome. Cambridge: National Down Syndrome Society, Library of Congress, Mit Press, 1988, p. 167-198.

7. Miller, J. F., Leddy, M., Leavitt, L. A. (eds.) Improving the Communication of People with Down Syndrome. Baltimore: Paul H. Brookes Publishing Company, 1999.

8. Kliewer, W., "Issues Involved in Facilitated Communication and People with Down Syndrome", in: Facilitated Communication Digest, vol. 3, n. 1, November 1994, pp 8-14. Online: http://www.altonweb.com/cs/downsyndrome/fcandds.html. Accessed on: 19-012001.

9. Spiker, D., Hopmann, M. R. "The effectiveness of Early Intervention for Children with Down Syndrome", in: Guaralnick, M. J. (Ed.) The effectiveness of Early Intervention, Chapter 13, Paul. H. Brookes Publishing Company, 1997. Online: http://www.altonweb. com/cs/downsyndrome/eieffective.html. Accessed on: 19-01-2001.

10.Vergason, G. A.; Anderegg, M. L. (Eds.). Dictionary of Special Education and Rehabilitation $4^{\mathrm{a}}$ ed. Denver: Love Publishing Company, 1997.

11.Von Tetzchner, S., Martinsen, H. Introdução à Comunicação Aumentativa e Alternativa. Porto: Porto Editora, Colecção Educação Especial, 2000.

12.Light, J. and Lindsay, P., Cognitive Science and Augmentative and Alternative Communication, Augmentative and Alternative Communication, 7, 1991, pp. 186-203.

13.Buckley, S. J., "Teaching children with Down syndrome to Read and Write", in: L. Nadel \& D. Rosenthal (Eds.), Down Syndrome: Living and Learning in the Community. New York: Wiley, 1995, pp. 158-169.

14. Barns, L., ASL - American Sign Language, New York, Harper Perennial, 1990.

15.SNRIPD, Gestuário de Língua Portuguesa, $2^{\mathrm{a}}$ ed., Lisboa, Secretariado Nacional de Reabilitação de Pessoas com Deficiência, 1995.

16. Le Prevost., P., See \& Say, T.F.H., Stourport, 1990.

17. Strauss, R., Managing Multimedia Projects, Boston, Focal Press, 1997. 